\title{
Invariance properties for coefficients of symmetric functions
}

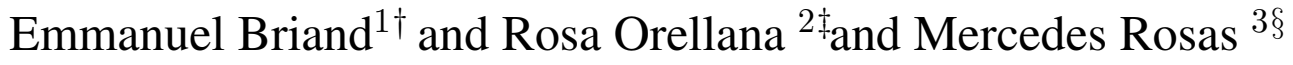 \\ ${ }^{1}$ Departamento de Matemática Aplicada I, Universidad de Sevilla, Avda. Reina Mercedes, 41012 Sevilla, Spain. \\ ${ }^{2}$ Dartmouth College, Mathematics Department, 6188 Kemeny Hall, Hanover, NH 03755, USA. \\ ${ }^{3}$ Departamento de Álgebra, Universidad de Sevilla, Avda. Reina Mercedes, 41012 Sevilla, Spain.
}

\begin{abstract}
We show that several of the main structural constants for symmetric functions (Littlewood-Richardson coefficients, Kronecker coefficients, plethysm coefficients, and the Kostka-Foulkes polynomials) share invariance properties related to the operations of taking complements with respect to rectangles and adding rectangles.

Résumé. Nous montrons que plusieurs des principales constantes de structure de la théorie des fonctions symétriques (les coefficients de Littlewood-Richardson, les coefficients de Kronecker, les coefficients du pléthysme, et les polynômes de Kostka-Foulkes) ont en commun des symétries décrites par des opérations de complémentation dans des rectangles et d'ajout de rectangles pour les partitions qui les étiquettent.
\end{abstract}

Keywords: Littlewood-Richardson coefficients, Kronecker coefficients, plethysm coefficients, Kostka coefficients

\section{Introduction}

This paper investigates some invariance properties of four of the main families of coefficients in the theory of symmetric functions: the Kostka numbers (and their deformations, the Kostka-Foulkes polynomials), the Littlewood-Richardson, the Kronecker, and the plethysm coefficients. These coefficients have applications to many different fields of mathematics such as representation theory, invariant theory and algebraic geometry, as well as physics and computer science. The presence of invariance relations often leads to a better understanding of the objects they enumerate, to simplifications in the number of cases in proofs, and in some cases, can be used to simplify computations.

In this paper we present a unified approach that shows some invariance relations for all these families of coefficients. These relations involve two operations on partitions: (i) taking complements in rectangles, or (ii) adding "tall" rectangles. We show that in the language of symmetric polynomials these symmetries

\footnotetext{
${ }^{\dagger}$ Email: ebriandeus.es. Partially supported by MTM2010-19336, MTM2013-40455-P, FQM-333, P12-FQM-2696 and FEDER.

‡Email: rosa.c.orellana@dartmouth. edu. Partially supported by NSF Grant DMS-130512

Email: mrosas@us.es. Partially supported by MTM2010-19336, MTM2013-40455-P, FQM-333, P12-FQM-2696 and FEDER.
} 
appear as evaluation at the inverses of the variables, for type (i), and factorization by the product of the variables, for type (ii).

We let $\square_{k, a}(\lambda)$ denote the complement of $\lambda$ with respect to a $k \times a$ rectangle, as illustrated in Figure 1

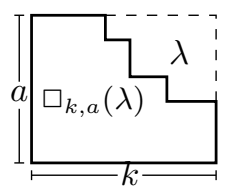

Fig. 1: The partition $\square_{k, a}(\lambda)$

For example in the case of the Littlewood-Richardson coefficients we have shown in Theorem 1 that they satisfy the following invariance relation with respect to taking complements in rectangles:

$$
c_{\lambda, \mu}^{\nu}=c_{\square_{l, n}(\lambda), \square_{m, n}(\mu)}^{\square_{l+m, n}(\nu)}, \quad \text { when } \lambda \subseteq\left(l^{n}\right), \mu \subseteq\left(m^{n}\right) \text { and } \nu \subseteq\left((l+m)^{n}\right)
$$

(see Figure 2). Moreover, in Theorem 2 we show the following invariance relation with respect to the operation of adding rectangles to the indexing partitions:

$$
c_{\lambda, \mu}^{\nu}=c_{\lambda+\left(k^{n}\right), \mu}^{\nu+\left(k^{n}\right)}, \quad \text { when } \nu \text { and } \lambda \text { have length at most } n .
$$
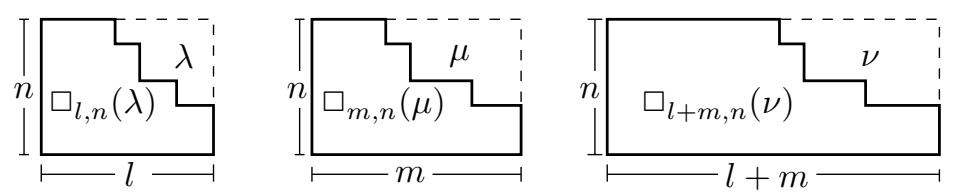

Fig. 2: The partitions occurring in the invariance relation of Theorem 1

Our aim is to show that analogous results hold for the other families of coefficients mentioned earlier. To prove our results we use some identities in the ring of Laurent symmetric functions. The details of our methods is discussed in Section 2.

\section{Algebraic tools}

We assume that the reader is familiar with the various algebraic structures on the space of symmetric functions, Sym, and in particular, with its main bases. For background information see [9, 13, 17]. We mainly follow the notation of [17], except for the fact that we draw our Ferrers diagrams using the French notation. Let $\mathcal{P}^{+}(n)$ be the set of all weakly decreasing sequences $\left(\lambda_{1}, \lambda_{2}, \ldots, \lambda_{n}\right)$ of nonnegative integers. When dealing with weakly decreasing sequences of integers, it will be convenient not to distinguish between sequences that differ only by trailing zeros. Therefore $\mathcal{P}^{+}(n)$ represents as well the set of integer partitions with length at most $n$. Given any two integer partitions $\lambda$ and $\mu, \lambda \subseteq \mu$ stands for the inclusion 
of the corresponding Ferrers diagrams, $\lambda^{\prime}$ is the conjugate of $\lambda$, and $\lambda+\mu$ is the partition whose parts are the $\lambda_{i}+\mu_{i}$. We use $\ell(\lambda)$ to denote the number of nonzero parts of $\lambda$, i.e., its length. Last, $\left(k^{n}\right)$ stands for the sequence with $n$ terms all equal to $k$.

Let $X=\left\{x_{1}, x_{2}, \ldots\right\}$ be a countable set of independent variables. For $n \geq 0$, we set $X_{n}=$ $\left\{x_{1}, x_{2}, \ldots, x_{n}\right\}$. The ring of symmetric polynomials, $\mathbb{Z}\left[x_{1}, x_{2}, \ldots, x_{n}\right]^{S_{n}}$, admits as a linear basis the Schur polynomials, $s_{\lambda}\left[X_{n}\right]=s_{\lambda}\left(x_{1}, x_{2}, \ldots, x_{n}\right)$, indexed by all $\lambda \in \mathcal{P}^{+}(n)$. They are defined by

$$
s_{\left(\lambda_{1}, \lambda_{2}, \ldots, \lambda_{n}\right)}[X]=\frac{\operatorname{det}\left(x_{i}^{\lambda_{j}+j-1}\right)_{1 \leq i, j \leq n}}{\operatorname{det}\left(x_{i}^{j-1}\right)_{1 \leq i, j \leq n}} .
$$

This is Jacobi's definition of Schur polynomials as "bialternants" [13, I.§3.(3.1)].

Let us consider now $\mathbb{Z}\left[x_{1}^{ \pm 1}, x_{2}^{ \pm 1}, \ldots, x_{n}^{ \pm 1}\right]^{S_{n}}$, the ring of symmetric Laurent polynomials in $n$ variables. Let $\mathcal{P}(n)$ be the set of all weakly decreasing sequences of integers $\left(\lambda_{1}, \lambda_{2}, \ldots, \lambda_{n}\right)$. (Compared to the definition of $\mathcal{P}^{+}(n)$, we dropped the requirement of nonnegativity). We define the Schur Laurent polynomials $s_{\lambda}\left[X_{n}\right]$, for $\lambda \in \mathcal{P}(n)$, again by (1). Denote by $X_{n} \vee$ the set of the inverses of the variables, i.e., $X_{n}{ }^{\vee}=\left\{\frac{1}{x_{1}}, \frac{1}{x_{2}}, \ldots, \frac{1}{x_{n}}\right\}$. For any sequence $\lambda \in \mathcal{P}(n)$ and any integer $k$, define the new sequence

$$
\square_{k, n}(\lambda)=\left(k-\lambda_{n}, k-\lambda_{n-1}, \ldots, k-\lambda_{1}\right) .
$$

This sequence is also in $\mathcal{P}(n)$. This extends the definition given in the introduction, when $\lambda$ is a partition that fits in the diagram of $\left(k^{n}\right)$. In that case, $\square_{k, n}(\lambda)$ is also a partition, "complement" of $\lambda$ in the rectangle. It is immediate to check from (1) the following properties:

Lemma 1. For all $\lambda \in \mathcal{P}(n)$ and all integers $k$, we have

$$
s_{\lambda+\left(k^{n}\right)}\left[X_{n}\right]=\left(x_{1} x_{2} \cdots x_{n}\right)^{k} s_{\lambda}\left[X_{n}\right]
$$

and

$$
s_{\lambda}\left[X_{n}^{\vee}\right]=s_{\square_{0, n}(\lambda)}\left[X_{n}\right] .
$$

Formula (3) is well known, see [9, (I.4.12.)], [17, Ex. 7.41] or [20, B].

Lemma 2. The Schur Laurent polynomials $s_{\lambda}\left[X_{n}\right]$, for $\lambda \in \mathcal{P}(n)$, are a basis for $\mathbb{Z}\left[x_{1}^{ \pm 1}, x_{2}^{ \pm 1}, \ldots, x_{n}^{ \pm 1}\right]^{S_{n}}$.

\section{Representation-theoretic interpretation of (3)}

Let $V$ be a complex vector space of dimension $n$. The Schur polynomials in $n$ variables are the formal characters of the irreducible polynomial representations of $G L(V)$, the $\mathbb{S}_{\lambda}(V)$ for $\lambda \in \mathcal{P}^{+}(n)$. The Schur Laurent polynomials are the formal characters of its rational irreducible representations.

Relation (2) corresponds to the isomorphism $\mathbb{S}_{\lambda+\left(k^{n}\right)}(V) \cong \mathbb{S}_{\lambda} \otimes D_{k}$, where $D_{k}$ is the one dimensional representation where $g \in G L(V)$ acts as the multiplication by $\operatorname{det}(g)^{k}$.

The Schur Laurent polynomial $s_{\lambda}\left[X_{n}{ }^{\vee}\right]$ is the formal character of the dual representation $\mathbb{S}_{\lambda}\left(V^{*}\right)$. The identity (3) means that $\mathbb{S}_{\lambda}\left(V^{*}\right) \cong \mathbb{S}_{\square_{0, n}(\lambda)}(V)$.

We will now exploit (2) and (3) systematically to produce symmetries for the Littlewood-Richardson coefficients, the Kronecker coefficients and the plethysm coefficients. In Section 6, we will extend (2) and (3) to Hall-Littlewood polynomials, to produce symmetries for the Kostka-Foulkes polynomials. 


\section{Littlewood-Richardson coefficients}

The Littlewood-Richardson coefficients are the structure constants in the ring of symmetric functions with respect to the basis of Schur functions.

$$
s_{\lambda} s_{\mu}=\sum_{\nu} c_{\lambda, \mu}^{\nu} s_{\nu}
$$

In representation theory, the Littlewood-Richardson coefficients describe the multiplicities of tensor products of irreducible representations of general linear groups, and also the multiplicities in the decompositions of certain induced representations of the symmetric group. In algebraic geometry, they are the structure coefficients when multiplying Schubert classes in the cohomology ring of the Grassmannian.

In this section we will prove the rectangular invariances for the Littlewood-Richardson coefficients. Let $n$ be a nonnegative integer and $\lambda$ and $\mu$ be two partitions. Specializing at $X_{n}=\left\{x_{1}, x_{2}, \ldots, x_{n}\right\}$, we get

$$
s_{\lambda}\left[X_{n}\right] s_{\mu}\left[X_{n}\right]=\sum_{\nu: \ell(\nu) \leq n} c_{\lambda, \mu}^{\nu} s_{\nu}\left[X_{n}\right]
$$

If $\ell(\lambda)$ or $\ell(\mu)$ is bigger than $n$, then the right-hand side is zero. Then all coefficients $c_{\lambda, \mu}^{\nu}$ in the righthand side are zero. We assume now that $\lambda$ and $\mu$ have length at most $n$. Let us replace each $x_{i}$ with $1 / x_{i}$. We obtain $s_{\lambda}\left[X_{n}{ }^{\vee}\right] s_{\mu}\left[X_{n}{ }^{\vee}\right]=\sum_{\nu: \ell(\nu) \leq n} c_{\lambda, \mu}^{\nu} s_{\nu}\left[X_{n}{ }^{\vee}\right]$. By [3], this can be written as

$$
s_{\square_{0, n}(\lambda)}\left[X_{n}\right] s_{\square_{0, n}(\mu)}\left[X_{n}\right]=\sum_{\nu: \ell(\nu) \leq n} c_{\lambda, \mu}^{\nu} s_{\square_{0, n}(\nu)}\left[X_{n}\right] .
$$

Let $l \geq \lambda_{1}$ and $m \geq \mu_{1}$. Let us multiply both sides with $\left(x_{1} x_{2} \cdots x_{n}\right)^{l+m}$. We get, by (2),

$$
s_{\square_{l, n}(\lambda)}\left[X_{n}\right] s_{\square_{m, n}(\mu)}\left[X_{n}\right]=\sum_{\nu: \ell(\nu) \leq n} c_{\lambda, \mu}^{\nu} s_{\square_{l+m, n}(\nu)}\left[X_{n}\right] .
$$

This implies the following theorem.

Theorem 1. Let $l, m, n$ be nonnegative integers and $\lambda, \mu$ and $\nu$ be three partitions such that $\ell(\nu) \leq n$, $\lambda_{1} \leq l$ and $\mu_{1} \leq m$. If $\lambda \subseteq\left(l^{n}\right), \mu \subseteq\left(m^{n}\right)$ and $\nu \subseteq\left((l+m)^{n}\right)$ then

$$
c_{\lambda, \mu}^{\nu}=c_{\square_{l, n}(\lambda), \square_{m, n}(\mu)}^{\square_{l+m, n}(\nu)}
$$

In the other cases, $c_{\lambda, \mu}^{\nu}=0$.

Note that the involution $\omega$ (that maps the elementary symmetric function $e_{i}$ to the complete sum $h_{i}$, see [13] I. $\$ 2$ ) yields an analogous invariance relation with respect to three rectangles of the same width, instead of height.

As an immediate consequence of Theorem 1, we obtain the following well-known identity for skew Schur functions. Let $\lambda \subseteq\left(m^{n}\right)$, then

$$
s_{\left(m^{n}\right) / \lambda}=s_{\left(m-\lambda_{m}, m-\lambda_{m-1}, \ldots, m-\lambda_{1}\right)} .
$$

In addition, by multiplying (4) and $\left(x_{1} x_{2} \cdots x_{n}\right)^{k}$ and using (2) we obtain the following translational invariance relation for $c_{\lambda, \mu}^{\nu}$. 
Theorem 2. Let $n \geq 0$ and $k$ be integers and $\lambda, \mu, \nu$ be partitions such that $n \geq \ell(\nu)$ and $\lambda+\left(k^{n}\right)$ is a partition (i.e. $\lambda_{n}+k \geq 0$ ). If $\ell(\lambda) \leq n$ and $\nu+\left(k^{n}\right)$ is a partition, then

$$
c_{\lambda, \mu}^{\nu}=c_{\lambda+\left(k^{n}\right), \mu}^{\nu+\left(k^{n}\right)}
$$

else $c_{\lambda, \mu}^{\nu}=0$.

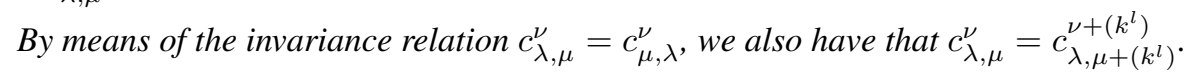

\section{Kronecker coefficients}

The Kronecker coefficients describe the multiplicities of tensor products of irreducible representations of symmetric groups, and also the multiplicities in the decompositions of certain induced representations of the general lineal group. Understanding the Kronecker coefficients is a major open problem in the representation theory of the symmetric and the general linear group. These coefficients also appear naturally in some interesting problems in quantum information theory [11, 12], geometric complexity theory [4, 2] and invariant theory.

In this section, we show how to use Formula (3) to derive a rectangular invariance relation for the Kronecker coefficients. We start with the following description of the Kronecker cofficients: let $X$ and $Y$ be two independent set of variables $x_{1}, x_{2}, \ldots$ and $y_{1}, y_{2}, \ldots$. Let $f[X Y]$ stand for the evaluation of the symmetric function $f$ at all products $x_{i} y_{j}$, this is a symmetric function in $X$ and in $Y$ and expands in the basis of the $s_{\lambda}[X] s_{\mu}[Y]$. Then, for all partitions $\nu$, (see [13, I. $\left.\S 7 .(7.9)\right]$ )

$$
s_{\nu}[X Y]=\sum_{\lambda, \mu} g(\lambda, \mu, \nu) s_{\lambda}[X] s_{\mu}[Y] .
$$

The similar proof as for the Littlewood-Richardson coefficients can be used to show the following rectangular and translational invariance relations for the Kronecker coefficients. For proofs and more details see [3].

Theorem 3. Let $l, m$ and $n$ be three nonnegative integers and $\lambda, \mu$ and $\nu$ be three partitions such that $\lambda_{1} \leq l, \mu_{1} \leq m, \nu_{1} \leq n$. If $\lambda \subseteq\left(l^{m n}\right), \mu \subseteq\left(m^{l n}\right)$ and $\nu \subseteq\left(n^{l m}\right)$, then

$$
g(\lambda, \mu, \nu)=g\left(\square_{l, m n}(\lambda), \square_{m, l n}(\mu), \square_{n, l m}(\nu)\right) .
$$

In the other cases, $g(\lambda, \mu, \nu)=0$.

Theorem 4. Let $\lambda, \mu$ and $\nu$ be partitions. Let $l \geq 0, m \geq 0$ and $k \in \mathbb{Z}$ be integers such that $l \geq \ell(\lambda)$, $m \geq \ell(\mu)$ and $\nu+\left(k^{l m}\right)$ is a partition (i.e. has no negative components). If $\ell(\nu) \leq \operatorname{lm}$ and $\lambda+\left((k m)^{l}\right)$ and $\mu+\left((k l)^{m}\right)$ are partitions, then

$$
g(\lambda, \mu, \nu)=g\left(\lambda+\left((k m)^{l}\right), \mu+\left((k l)^{m}\right), \nu+\left((k)^{l m}\right)\right)
$$

and else $g(\lambda, \mu, \nu)=0$.

An important class of Kronecker coefficients are those indexed by rectangular partitions. They are important in quantum information theory to model entanglement [11,12] and also to advance the program of Geometric Complexity Theory [4]. As a corollary we easily recover the result of Stembridge [20, (C.1)]. 
Corollary 1. Let $k$ and $d$ be nonnegative integers. If $k \leq d^{2}$,

$$
g\left(\left(d^{k}\right),\left(d^{k}\right),\left(d^{k}\right)\right)=g\left(\left(d^{d^{2}-k}\right),\left(d^{d^{2}-k}\right),\left(d^{d^{2}-k}\right)\right)
$$

and when $k>d^{2}$, this Kronecker coefficient is zero.

\section{Weight reduction for Kronecker coefficients}

The naive algorithm to compute a Kronecker coefficient $g(\lambda, \mu, \nu)$ consists in converting Schur functions in power sums. Indeed, in the power sums basis, Kronecker products are trivial. This is the algorithm used, for instance, currently in SAGE [18] and the Maple package SF [19]. The cost of the computation depends then mainly on the weight of $\lambda, \mu$ and $\nu$. (Note that other algorithms are available and efficient for partitions of short height, regardless of the weight, see for instance [6]).

Theorem 3 shows that $g(\lambda, \mu, \nu)$ is equal to other Kronecker coefficients, that may be of smaller weight. Precisely, let $N=|\lambda|=|\mu|=|\nu|$. Then the weight of $g\left(\square_{l, m n}(\lambda), \square_{m, l n}(\mu), \square_{n, l m}(\nu)\right)$ (i.e. the weight of the indexing partitions) is $l m n-N$. We can take $l=\lambda_{1}, m=\mu_{1}$ and $n=\nu_{1}$, the computation is reduced to the computation of a Kronecker coefficient of weight $\lambda_{1} \mu_{1} \nu_{1}-N$. Last, we may make use of the symmetries under conjugation $g(\lambda, \mu, \nu)=g\left(\lambda, \mu^{\prime}, \nu^{\prime}\right)=g\left(\lambda^{\prime}, \mu, \nu^{\prime}\right)=g\left(\lambda^{\prime}, \mu^{\prime}, \nu\right)$ to reduce the computation to the computation of a Kronecker coefficient whose weight is the smallest among

$$
K \frac{\lambda_{1}}{\ell(\lambda)}-N, \quad K \frac{\mu_{1}}{\ell(\mu)}-N, \quad K \frac{\nu_{1}}{\ell(\nu)}-N, \quad \text { and } K \frac{\lambda_{1} \mu_{1} \nu_{1}}{\ell(\lambda) \ell(\mu) \ell(\nu)}-N
$$

\section{Representation-theoretic interpretation of Corollary 1.}

Let $V$ be a complex vector space of dimension $d$. Consider the exterior algebra: $\Lambda(V \otimes V \otimes V)=$ $\bigoplus_{i=0}^{d^{3}} \Lambda^{i}(V \otimes V \otimes V)$. The group $G L(V) \times G L(V) \times G L(V)$ acts on this exterior algebra. The Kronecker coefficient $g\left(\lambda^{\prime}, \mu^{\prime}, \nu^{\prime}\right)$ is the multiplicity of its irreducible representation $\mathbb{S}_{\lambda}(V) \otimes \mathbb{S}_{\mu}(V) \otimes \mathbb{S}_{\nu}(V)$. In particular, $\Lambda^{i}(V \otimes V \otimes V)$ contains non-trivial invariants for $S L(V) \times S L(V) \times S L(V)$ only if there exists an integer $k$ such that $i=k d$. Then the dimension of the subspace of invariants is the rectangular Kronecker coefficient $g\left(\left(d^{k}\right),\left(d^{k}\right),\left(d^{k}\right)\right)$. Equation (5) follows from the $S L(V \otimes V \otimes V)$ natural isomorphism $\Lambda^{i}\left(V^{*} \otimes V^{*} \otimes V^{*}\right) \cong \Lambda^{d^{3}-i}(V \otimes V \otimes V)$.

\section{Plethysm coefficients}

The plethysm of two symmetric functions $f$ and $g$ is denoted by $f[g]$. This operation was introduced by Littlewood [10] in the context of compositions of representations of the general linear groups. Plethysm has important applications to physics [23] and invariant theory [7]. The plethysm coefficients are the coefficients $a_{\lambda, \mu}^{\nu}$ of the plethysm of two Schur functions, expanded in the Schur basis:

$$
s_{\lambda}\left[s_{\mu}\right]=\sum_{\nu} a_{\lambda, \mu}^{\nu} s_{\nu}
$$

While there are algorithms for computing $a_{\lambda, \mu}^{\nu}$ (see for example [5, 24]), no satisfying combinatorial description has been found. In this section we describe two rectangular symmetries satisfied by the plethysm coefficients. 

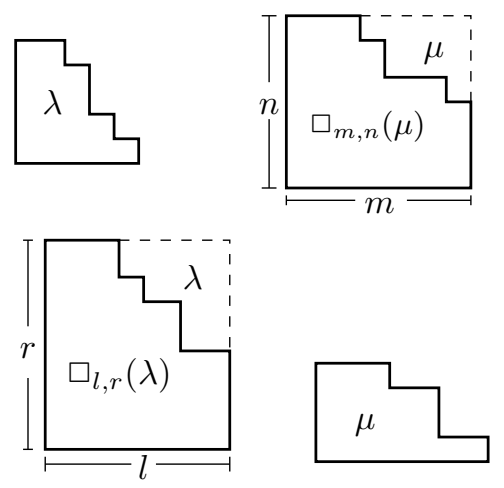
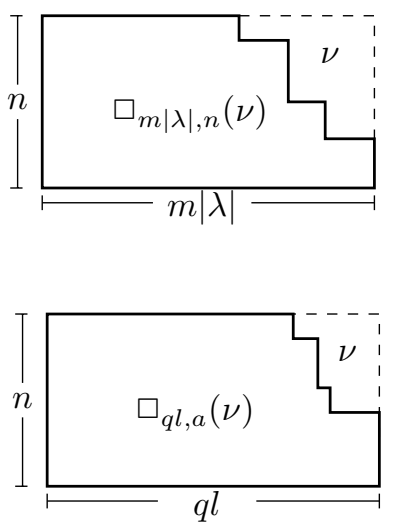

Fig. 3: The partitions in the invariance relation of Theorem 5 (top) and Theorem 7 (bottom)

It will be useful to extend the plethysm operation to the case when $f$ is a symmetric function but $g=g\left(x_{1}, x_{2}, \ldots\right)$ is any formal series. This is done by means of the following two rules: (1) the map $f \mapsto f[g]$ is a morphism of algebras; and (2) for any positive integer $n, p_{n}[g]=g\left(x_{1}^{n}, x_{2}^{n}, \ldots\right)$ (here $p_{n}$ is the $n$-th power sum symmetric function).

This determines $f[g]$ for any symmetric function $f$, since the algebra of symmetric functions with rational coefficients is freely generated by the power sums $p_{n}$. When $g$ is a symmetric function, $f[g]$ defined as above coincides with the plethysm of $f$ with $g$, see [13, I. $\S 8$.] or [17, Def. A.2.6]. We will make use of the following property.

Lemma 3. Let $f$ be a homogeneous symmetric function of degree $L$ and $g\left(x_{1}, x_{2}, \ldots\right)$ be a formal series. Let $x^{w}$ be a monomial in $x$. Then $f\left[x^{w} g\right]=x^{L w} f[g]$.

\section{First pair of symmetries for plethysm coefficients}

Let $X_{n}=\left\{x_{1}, x_{2}, \ldots, x_{n}\right\}$ be a set of $n$ variables, where $n$ is a nonnegative integer, and let $\lambda$ and $\mu$ be two partitions. Evaluating (5) at $X_{n}$ we get $s_{\lambda}\left[s_{\mu}\left[X_{n}\right]\right]=\sum_{\nu: \ell(\nu) \leq n} a_{\lambda, \mu}^{\nu} s_{\nu}\left[X_{n}\right]$. Applying similar ideas as for the Littlewood-Richardson and Kronecker coefficients we obtain the following symmetries for plethysm coefficients, for details see [3].

Theorem 5. Fix nonnegative integers $m$ and $n$ and let $\lambda, \mu$ and $\nu$ be partitions such that $\mu \subseteq\left(m^{n}\right)$ and $\ell(\nu) \leq n$. If $\nu \subseteq\left((m|\lambda|)^{n}\right)$, then

$$
a_{\lambda, \mu}^{\nu}=a_{\lambda, \square_{m, n}(\mu)}^{\square_{m|\lambda|, n}(\nu)}
$$

Otherwise $a_{\lambda, \mu}^{\nu}=0$.

Theorem 6. Let $\lambda, \mu$ and $\nu$ be partitions. Let $n \geq 0$ and $k \in \mathbb{Z}$ be integers such that $\ell(\nu) \leq n$ and $\mu+\left(k^{n}\right)$ is a partition.

If $\nu+\left((k|\lambda|)^{n}\right)$ is a partition, then

$$
a_{\lambda, \mu}^{\nu}=a_{\lambda, \mu+\left(k^{n}\right)}^{\nu+\left((k|\lambda|)^{n}\right)}
$$


and else $a_{\lambda, \mu}^{\nu}=0$.

\section{Second pair of symmetries for plethysm coefficients}

There is another way to exploit the alphabet of inverses for plethysm coefficients in order to obtain another rectangular invariance relation. Recall that the combinatorial definition of Schur functions says that,

$$
s_{\mu}=\sum_{T} x^{w(T)}
$$

where the sum is carried over all semistandard tableaux $T$ of shape $\mu$. The exponent $w(T)$ is the weight of $T$, i.e., its $i$-th component is the number of occurrences of $i$ in $T$. For details see [17, §7.10.1.].

If $f$ is a symmetric function and $g$ a sum of monomials, then $f[g]$ is the specialization of $f$ at these monomials [13, I. $\S 8]: f\left[x^{\omega_{1}}+x^{\omega_{2}}+\ldots\right]=f\left(x^{\omega_{1}}, x^{\omega_{2}}, \ldots\right)$. This holds in particular for $g=s_{\mu}[X]$.

A careful analysis of the evaluation at the alphabet of inverses leads to the following results. See [3] for further details.

Theorem 7. Let $l$ and $n$ be nonnegative integers and $\mu, \nu$, and $\lambda$ be partitions such that $\lambda_{1} \leq l$, and $\ell(\nu) \leq n$. Let $r$ be the number of semistandard tableaux of shape $\mu$ and entries in $\{1,2, \ldots, n\}$. Then $q=r|\mu| / n$ is an integer, and we have that if $\lambda \subseteq\left(l^{r}\right)$ and $\nu \subseteq\left((q l)^{n}\right)$,

$$
a_{\lambda, \mu}^{\nu}=a_{\square, r, r}^{\square_{q l, n}(\nu), \mu},
$$

and otherwise $a_{\lambda, \mu}^{\nu}=0$.

Theorem 8. Let $\lambda, \mu$ and $\nu$ be partitions. Let $n \geq 0$ and $k \in \mathbb{Z}$ be integers such that $\ell(\nu) \leq n$. Let $r$ and $q$ be defined as in Theorem 7 Assume that $\lambda+\left(k^{r}\right)$ is a partition. If $\ell(\lambda) \leq r$ and $\nu+\left((q k)^{n}\right)$ is a partition, then

$$
a_{\lambda, \mu}^{\nu}=a_{\lambda+\left(k^{r}\right), \mu}^{\nu+\left((q)^{n}\right)},
$$

else $a_{\lambda, \mu}^{\nu}=0$.

Remark. The number $r=\# \mathcal{T}_{\mu}(n)$ is given by the hook-content formula (see [17 pg. 376]).

\section{Weight reduction for Plethysm coefficients}

As for Kronecker products (see 4), plethysms are trivial in the basis of power sums. Plethysm coefficients can thus be computed by means of conversions to the power sums basis (this is done this way in SAGE and SF [18, 19]). When performing such a computation, it is very helpful to reduce the weight of the symmetric functions involved. This can be done, in some cases, by means of Theorem 5 The weight for the plethysm coefficient $a_{\lambda, \mu}^{\nu}$ is $N=|\nu|=|\lambda| \cdot|\mu|$. Theorem 5 shows that this plethysm coefficient is equal to another plethysm coefficient with weight $\mu_{1} \ell(\nu)|\lambda|-N$. We can also make use of the symmetries $([13$, I. $\S 8 . E x .1(a)])$

$$
a_{\lambda, \mu}^{\nu}=\left\{\begin{array}{l}
a_{\lambda, \mu^{\prime}}^{\nu^{\prime}} \text { when }|\mu| \text { is even, } \\
a_{\lambda^{\prime}, \mu^{\prime}}^{\nu^{\prime}} \text { when }|\mu| \text { is odd. }
\end{array}\right.
$$

Set $K=\ell(\mu) \ell(\nu)|\lambda|$. We can obtain, therefore, a reduction to the weight: $K \cdot \min \left(\frac{\mu_{1}}{\ell(\mu)}, \frac{\nu_{1}}{\ell(\nu)}\right)-N$. 


\section{Kostka-Foulkes polynomials}

The Kostka-Foulkes polynomials $K_{\lambda, \mu}(t)$ are the coefficients that appear in the decompositions of Schur functions in the basis of Hall-Littlewood polynomials $P_{\mu}(X ; t)$ :

$$
s_{\lambda}[X]=\sum_{\mu} K_{\lambda, \mu}(t) P_{\mu}(X ; t) .
$$

They are deformations of the Kostka numbers. We recover the Kostka numbers by evaluating the KostkaFoulkes polynomials at $t=1$.

On the other hand, their specialization at a finite set of variables $X_{n}=\left\{x_{1}, x_{2}, \ldots, x_{n}\right\}$, with $n \geq \ell(\mu)$ is given [13, III.(2.1)] by

$$
P_{\mu}\left(X_{n} ; t\right)=\frac{1}{v_{\mu, n}(t)} \sum_{w \in S_{n}} w\left(x_{1}^{\mu_{1}} \cdots x_{n}^{\mu_{n}} \prod_{i<j} \frac{x_{i}-t x_{j}}{x_{i}-x_{j}}\right) .
$$

where $w \in S_{n}$ permutes the variables $x_{i}$ and $v_{\mu, n}(t)=\prod_{i} \prod_{r=1}^{m_{i}(\mu)} \frac{1-t^{r}}{1-t}$, and $m_{i}$ is the number of occurrences of $i$ in the sequence $\mu$, once it has been padded with zeros to get length $n$.

As in the case of Schur polynomials, this definition still makes sense perfectly when $\mu \in \mathcal{P}(n)$ (with possible negative coordinates). We get the following generalization of (3):

Lemma 4. Let $\mu$ be a weakly decreasing sequence of integers, of length $n$, and $X=\left\{x_{1}, x_{2}, \ldots, x_{n}\right\}$. We have

$$
P_{\mu}\left(X_{n}^{\vee} ; t\right)=P_{\square_{0, n}(\mu)}\left(X_{n} ; t\right)
$$

and for any integer $k$,

$$
P_{\mu+\left(k^{n}\right)}\left(X_{n} ; t\right)=\left(x_{1} x_{2} \cdots x_{n}\right)^{k} \cdot P_{\mu}\left(X_{n} ; t\right) .
$$

Using Lemma 4 and (6) we get the following results.

Theorem 9. Let $k$ and $n$ be nonnegative integers.

Let $\lambda$ and $\mu$ be partitions such that $\lambda_{1} \leq k$ and $\ell(\mu) \leq n$. If $\lambda \subseteq\left(k^{n}\right)$ and $\mu \subseteq\left(k^{n}\right)$ then

$$
K_{\lambda, \mu}(t)=K_{\square_{k, n}(\lambda), \square_{k, n}(\mu)}(t) .
$$

Else $K_{\lambda, \mu}(t)=0$.

Specializing (5) at $X_{n}$ and multiplying with $\left(x_{1} x_{2} \cdots x_{n}\right)^{k}$, and using (2) and (6), we get the following result.

Theorem 10. Let $n$ and $k$ be integers, with $n \geq 0$. Let $\lambda$ and $\mu$ be partitions, with $\ell(\mu) \leq n$ and such that $\lambda+\left(k^{n}\right)$ is a partition.

If $\ell(\lambda) \leq n$ and $\mu+\left(k^{n}\right)$ is a partition then

$$
K_{\lambda, \mu}(t)=K_{\lambda+\left(k^{n}\right), \mu+\left(k^{n}\right)}(t),
$$

else $K_{\lambda, \mu}(t)=0$. 


\section{Final Remarks}

1. All the symmetries described in this extended abstract involve the operations of (i) taking complements in rectangles, or (ii) adding "tall" rectangles to the parts. They can also be shown to hold using the language of representation theory. The symmetries of type (i) actually follow from duality between representations of general linear groups, and those of type (ii) from factoring by determinant representations.

2. Other invariances for the Littlewood-Richardson coefficients, such as $c_{\lambda, \mu}^{\nu}=c_{\mu, \lambda}^{\nu}$ and $c_{\lambda, \mu}^{\nu}=$ $c_{\lambda, \square_{m, n}(\nu)}^{\square_{m, n}(\mu)}$, have been extensively studied. In particular there are several bijective proofs for them, see [14, 15, 21] and the references therein. These other invariances, that generate a full symmetric group $S_{3}$, are also obvious in the setting of Schubert calculus, since the numbers $c_{\lambda, \nu}^{\square_{m, n}(\mu)}$ interpret as triple intersections of Schubert varieties [8, §4, Eq. (23)]. We believe that it would be interesting to find a bijective proof of Theorem 1

3. The invariance relation presented in Theorem 1 is probably folklore, but we did not find it in the literature in the way presented here. It is, however, equivalent to the invariance relation mentioned in [1, $\S 2$. rem.(a)], as the generator of the $\mathbb{Z}_{2}$ subgroup in a $\mathbb{Z}_{2} \times S_{3}$ group of symmetries (the factor $S_{3}$ is the group of other invariances mentioned above in Remark 2).

4. The identity presented in Theorem 2 is very easily established from the combinatorial descriptions of the Littlewood-Richardson coefficients (e.g. the Littlewood-Richardson rule). The analogous identities described for Kronecker and plethysm coefficients, for which akin combinatorial descriptions are unavailable, are then more difficult to prove without Schur polynomials (or equivalent representation-theoretic considerations).

5. The invariance relation described in (3) is equivalent to the one found by Stembridge [20]. The method is basically the same, except for the presentation: where Stembridge uses representations of general linear groups we use their formal characters (symmetric Laurent polynomials). Our version is slightly more symmetric.

6. The translational invariance for the Kronecker coefficients described in Theorem 4 is well-known. It appears, for instance in [22, Theorem 3.1], [2, Lemma 2.1].

7. The bijection in the solution of exercise 7.41 in [17] gives a bijective proof for the specialization of Theorem 9 to the Kostka numbers. But this does not generalize to a bijective proof for the identity for the Kostka-Foulkes polynomials. The autors would like to see a bijective proof of this result.

8. The invariance relation described in Theorem 9 also follows from a much more elaborate result by Shimozono and Weyman on the Poincare polynomials of graded characters of isotopic components of a natural family of $G L\left(\mathbb{C}^{n}\right)$-modules supported in the closure of a nilpotent conjugacy class [16, Eq. (2.16)]. 
9. The Macdonald polynomials $P_{\lambda}\left(X_{n} ; q, t\right)$, defined in [13] Ch. VI, satisfy the relation

$$
\left(x_{1} \cdots x_{n}\right)^{k} P_{\lambda}\left(X^{\vee} ; q, t\right)=P_{\square_{k, n}(\lambda)}(X ; q, t) .
$$

Hence one can obtain similar relations for coefficients related to these basis. The results of this investigation will appear as a separate note.

\section{Acknowledgments}

The authors want to thank J. Stembridge for sending us his preprints, and to R. King, F. Bergeron, and O. Azenhas for helpful conversations. They also thank M. Zabrocki for his help in understanding how these invariances generalize to Macdonald polynomials. R. Orellana is grateful for the hospitality of the University of Sevilla and IMUS. The authors thank two anonymous referees for carefully reading the manuscript and for providing many detailed comments to help with the exposition.

\section{References}

[1] A. D. Berenstein and A. V. Zelevinsky, Triple multiplicities for $\operatorname{sl}(r+1)$ and the spectrum of the exterior algebra of the adjoint representation, J. Algebraic Combin. 1 (1992), no. 1, 7-22. MR 1162639 (93h:17012)

[2] Emmanuel Briand, Rosa Orellana, and Mercedes Rosas, Reduced Kronecker coefficients and counter-examples to Mulmuley's strong saturation conjecture SH, Comput. Complexity 18 (2009), no. 4, 577-600, With an appendix by Ketan Mulmuley. MR 2570451 (2011d:68055)

[3] _ Rectangular symmetries for coefficients of symmetric functions, http://arxiv.org/pdf/1410.8017v1.pdf, 2014.

[4] Peter Bürgisser, J. M. Landsberg, Laurent Manivel, and Jerzy Weyman, An overview of mathematical issues arising in the Geometric complexity approach to VP vs. VNP, SIAM J. Comput. 40 (2011), no. 4, 1179-1209.

[5] Y. M. Chen, A. M. Garsia, and J. Remmel, Algorithms for plethysm, Combinatorics and algebra (Boulder, Colo., 1983), Contemp. Math., vol. 34, Amer. Math. Soc., Providence, RI, 1984, pp. 109153.

[6] Matthias Christandl, Brent Doran, and Michael Walter, Computing multiplicities of lie group representations, 53rd Annual IEEE Symposium on Foundations of Computer Science, FOCS 2012, New Brunswick, NJ, USA, October 20-23, 2012, 2012, pp. 639-648.

[7] H. O. Foulkes, Concomitants of the quintic and sextic up to degree four in the coefficients of the ground form, J. London Math. Soc. 25 (1950), 205-209.

[8] William Fulton, Eigenvalues, invariant factors, highest weights, and Schubert calculus, Bull. Amer. Math. Soc. (N.S.) 37 (2000), no. 3, 209-249 (electronic). MR 1754641 (2001g:15023)

[9] Alain Lascoux, Symmetric functions and combinatorial operators on polynomials, CBMS Regional Conference Series in Mathematics, vol. 99, American Mathematical Society, Providence, RI, 2003. 
[10] D. E. Littlewood, Invariant theory, tensors and group characters, Philos. Trans. Roy. Soc. London Ser. A 239 (1944), 305-365.

[11] Jean-Gabriel Luque and Jean-Yves Thibon, Polynomial invariants of four qubits, Phys. Rev. A (3) 67 (2003), no. 4, 042303, 5. MR 2039690 (2004k:81098)

[12] _ Algebraic invariants of five qubits, J. Phys. A 39 (2006), no. 2, 371-377. MR 2198966 (2006h:81048)

[13] I. G. Macdonald, Symmetric functions and Hall polynomials, second ed., Oxford Mathematical Monographs, The Clarendon Press, Oxford University Press, New York, 1995.

[14] Igor Pak and Ernesto Vallejo, Combinatorics and geometry of Littlewood-Richardson cones, European J. Combin. 26 (2005), no. 6, 995-1008. MR 2143205 (2006e:05187)

[15] _ Reductions of Young tableau bijections, SIAM J. Discrete Math. 24 (2010), no. 1, 113-145. MR 2600656 (2011e:05278)

[16] Mark Shimozono and Jerzy Weyman, Graded characters of modules supported in the closure of a nilpotent conjugacy class, European J. Combin. 21 (2000), no. 2, 257-288. MR 1742440 (2002b:05136)

[17] Richard Stanley, Enumerative combinatorics, Cambridge Studies in Advanced Mathematics, 62, vol. 2, Cambridge University Press, 1999.

[18] W. A. Stein et al., Sage Mathematics Software (Version 6.3), The Sage Development Team, 2014, http://www.sagemath.org.

[19] John R. Stembridge, The SF Maple Package, version 2.4, 2005, http://www.math.1sa. umich.edu/ jrs/maple.html\#SF.

[20] __ Appendix to: Generalized stability of Kronecker coefficients, Preprint, available at http: //www.math.lsa.umich.edu/ jrs/papers/kron-app.pdf (consulted sept. 29, 2014)., August 2014.

[21] Hugh Thomas and Alexander Yong, An $S_{3}$-symmetric Littlewood-Richardson rule, Math. Res. Lett. 15 (2008), no. 5, 1027-1037. MR 2443999 (2009h:05213)

[22] Ernesto Vallejo, A stability property for coefficients in Kronecker products of complex $S_{n}$ characters, Electron. J. Combin. 16 (2009), no. 1, Note 22, 8. MR 2529813 (2011b:20040)

[23] Brian G. Wybourne, Symmetry principles and atomic spectroscopy, Wiley-Interscience [A division of John Wiley \& Sons], New York-London-Sydney, 1970, Including an appendix of tables by P. H. Butler. MR 0421392 (54 \#9396)

[24] Mei Yang, An algorithm for computing plethysm coefficients, Proceedings of the 7th Conference on Formal Power Series and Algebraic Combinatorics (Noisy-le-Grand, 1995), vol. 180, 1998, pp. 391402. MR 1603696 (99d:05088) 